\title{
Effects of Vinyltriethoxysilane and Maleic Anhydride Grafted Polypropylenes on the Morphological, Thermal, Rheological, and Mechanical Properties of Polypropylene/Clay Nanocomposites ${ }^{\dagger}$
}

\author{
Heriarivelo Risite ${ }^{1, *}$, Hicham Abou Oualid ${ }^{2}$ and Khalil ElMabrouk ${ }^{3}$ \\ 1 Département de Physique de l’Université de Toliara, Toliara 601, Madagascar \\ 2 Faculty of Sciences and Technologies, University Hassan II of Casablanca, Mohamedia, Mohammedia B. P. \\ 146, 20650, Morocco;Hicham.abououalid@gmail.com \\ 3 Euromed Research Center, Euromed University of Fes, Eco-Campus, Meknes Road, 30 300, Fes, Morocco; \\ k.elmabrouk@ueuromed.org \\ * Correspondence: h.risite@gmail.com \\ † Presented at the 1st International Online Conference on Nanomaterials, 1-15 September 2018; Available \\ online: https://iocn-2018-1.sciforum.net/.
}

Published: 5 September 2018

\begin{abstract}
The morphology and properties of polypropylene (PP)/organoclay nanocomposites prepared by melt processing were investigated with a special interest on the different effects of the use of different grafted PP as compatibilizers, i.e., maleic anhydride or silane-grafted species, PP-g-MA or PP-g-Si. When either PP-g-MA or PP-g-Si was added, better improvement of properties was achieved. The addition of PP-g-Si was found to increase the crystallization temperature upon the clay addition in comparison to PP-g-MA. Moreover, the PP-g-MA proved to be more efficient than PP-g-Si. The degree of reinforcement was found to be dependent on the interaction forces between the polymer matrix/clay, which resulted in intercalated/partial exfoliated structures for PP-g-Si while increasing clay content induced a change from exfoliated to intercalated using PP-g-MA, as revealed by transmission electron microscopy observations and X-ray diffraction analysis.
\end{abstract}

Keywords: nanocomposites; reinforcements; polypropylene functionalization; thermal properties; mechanical properties; rheology

\section{Introduction}

Filler-reinforced polymeric nanocomposite systems with well-dispersed inorganic nanoparticles have a tendency to exhibit significant improvements in physical and mechanical properties over their neat resin counterpart. The most commonly produced nanocomposite systems are polymer-layered silicate nanocomposites, which are of interest because of their exceptional reinforcement effects at very low loading. This characteristic has been exploited to prepare commercially viable structural components since minimized nanofiller loading results in a lighter structure, good processability, and increased ductility [1].

Polypropylene (PP)/clay nanocomposites have found many applications in industry. However, preparing well dispersed nanofiller in polymer matrix can pose a challenge during processing due to the difference in the polarity of nonpolar PP versus polar filler, which can lead to weak interfacial adhesion between polymer matrix and filler. To overcome this problem, a wide variety of polar groups, such as maleic anhydride (MA), glycidyl methacrylate, acrylic acid, diethyl maleate, butyl acrylate, and polyallyl, have been used to achieve compatibilization and good 
dispersion, by grafting these molecules onto a polymer backbone [2-5]. This is expected to enhance the polarity of the PP, followed by the interfacial adhesion between polymer and filler.

Recently, vinylakoxysilanes, such as vinyltrimethoxysilane (VTMS) and vinyltriethoxysilane (VTES), are used in the manufacturing of crosslinkable polyolefin. The two functional groups of vinyl group and alkoxy can, respectively, be grafted on a PP backbone and be hydrolyzed to generate silanols that can be coupled with each other to form crosslinkage [6-13]. However, this technique may present problems on the final products because excess crosslinking loading could create defects at the shaping materials. In this regard, VTES grafted PP (PP-g-Si) was used and it was found that appropriate graft contents and the efficiencies of moisture-curing provided favorable rheological properties without attending the gel point [14]. It can thus be expected that the use of PP-g-Si as compatibilizer can cause an enhancement in the interaction between PP and clay by controlling the method of preparation.

The use of such molecules showed an improvement in the final properties of the resulting nanocomposites, however, the study of the degree of reinforcement of each molecule on one grade type of polymer (PP or polyethylene, PE) in the presence of the clay is not well documented. This would allow assessment of the efficiency of each molecule compared to the other on a given polymer and permit an understanding of the parameters governing the property improvements. Contact angle measurement was used as a useful technique to measure the wettability for evaluation of compatibilizer species (i.e., isotactic homopolymer, MA grafted, and silane-grafted species) for the synthesis of a nanocomposite [15]. The maleated polymer and the organophilic coated clay were found to provide complete wetting, making the system an ideal candidate for subsequent intercalation. However, the experiments showing the difference in terms of properties have not been done

Based on these points of view, in the present study, PP was functionalized by peroxide-initiated grafting of MA and VTES to obtain PP-g-MA and PP-g-Si derivatives, respectively, to prepare nanocomposites. The effects of the usage of the compatibilizers on the prepared nanocomposites were studied and assessed by X-ray diffraction (XRD), transmission electronic microscopy (TEM), thermogravimetric analysis (TGA), rheometry in small amplitude oscillatory shear, and mechanical testing.

\section{Results and Discussions}

\subsection{Structural Characterizations}

Figure 1 and 2 shows a series of XRD patterns of all nanocomposites, including virgin clay. C20A clay showed a peak centered at $2 \theta=3.6^{\circ}$, corresponding to the basal plane peak doo1 of $2.45 \mathrm{~nm}$. For nanocomposites compatibilized with $\mathrm{PP}$-g-Si, it was difficult to determine the interlayer spacing due to the peak broadening in comparison to the C20A peak. However, it should be noted that the peak characteristic of the interlayer spacing was present at a small quantity, (around $2 \theta=3.1^{\circ}$, corresponding to d 001 of $2.94 \mathrm{~nm}$ ). The decrease in intensity and the broadening of the peak is probably due to the disorder generated by the stacks of layered silicates while maintaining a periodic distance and to the partial exfoliation of layered silicates. TEM observations of selected nanocomposites, as shown in Figure 3, supported these assumptions. The microscopy confirmed that PP is intercalated into the interlayer spacing of clay galleries. To evaluate the d-spacing of intercalated PP-g-Si nanocomposites, the distance was also calculated within image J software and by using TEM images of intercalated PP/PP-g-Si/C20A3 recorded at higher magnifications (Figure 4). TEM observations were found to be in good concordance with $\mathrm{XRD}$ and the calculated interlayer spacing was found to be around $2.91 \mathrm{~nm}$. However, using PP-g-MA, the interlayer characteristic peak appeared only at high clay loadings $(8 \mathrm{wt} \%)$, which confirmed the complete exfoliation at clay loadings $\leq 5 \mathrm{wt} \%$ whereas the coexistence of partial exfoliated and intercalated structures were obtained at $8 \mathrm{wt} \%$. To explain this difference, the graft content and also the amplitude of polarity between VTES and MA are supposed to be responsible. In the case of PP-g-Si, and during melt extrusion, the alkylammoniumbased clay has the same aliphatic apolar nature as the ethyl end groups of silane grafted onto the PP backbone (in another word, the silane grafted interacts with silicates via Van der Walls bonds, bonds 
of low energy) that induce the formation of the stacked structure after cessation of shear. In the case of PP-g-MA, it was easier to create hydrogen bonding with silicates (bonds of higher energy than Van der Walls bonds [16]), therefore, when equilibrium was reached, there as a sufficient excess enthalpy to promote an exfoliated nanocomposite structure. In fact, in the melt, the mass transport of the polymer entering the interlayer space is fast, and the polymer chains exhibit a mobility similar or faster than the self-diffusion [17]. If thermodynamic conditions are favorable for intercalation, the polymer can crawl in and out of the interlayer space until equilibrium is reached.

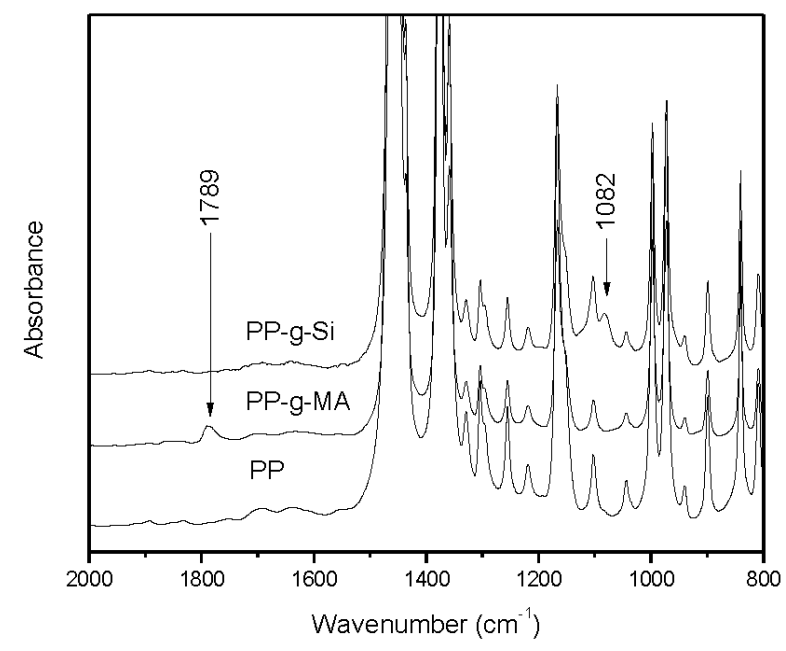

Figure 1. FTIR spectra of PP, PP-g-Si, and PP-g-MA.

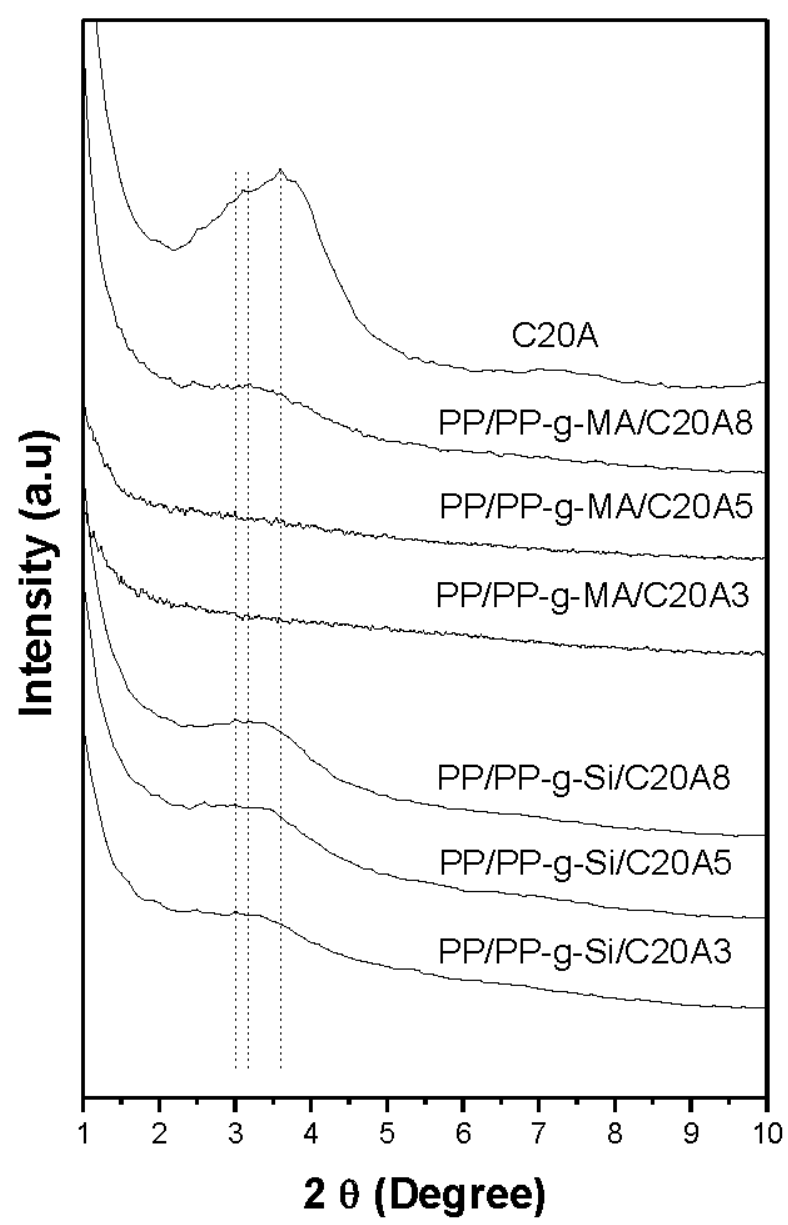

Figure 2. XRD patterns for the clays and PP nanocomposites at different clay contents. 

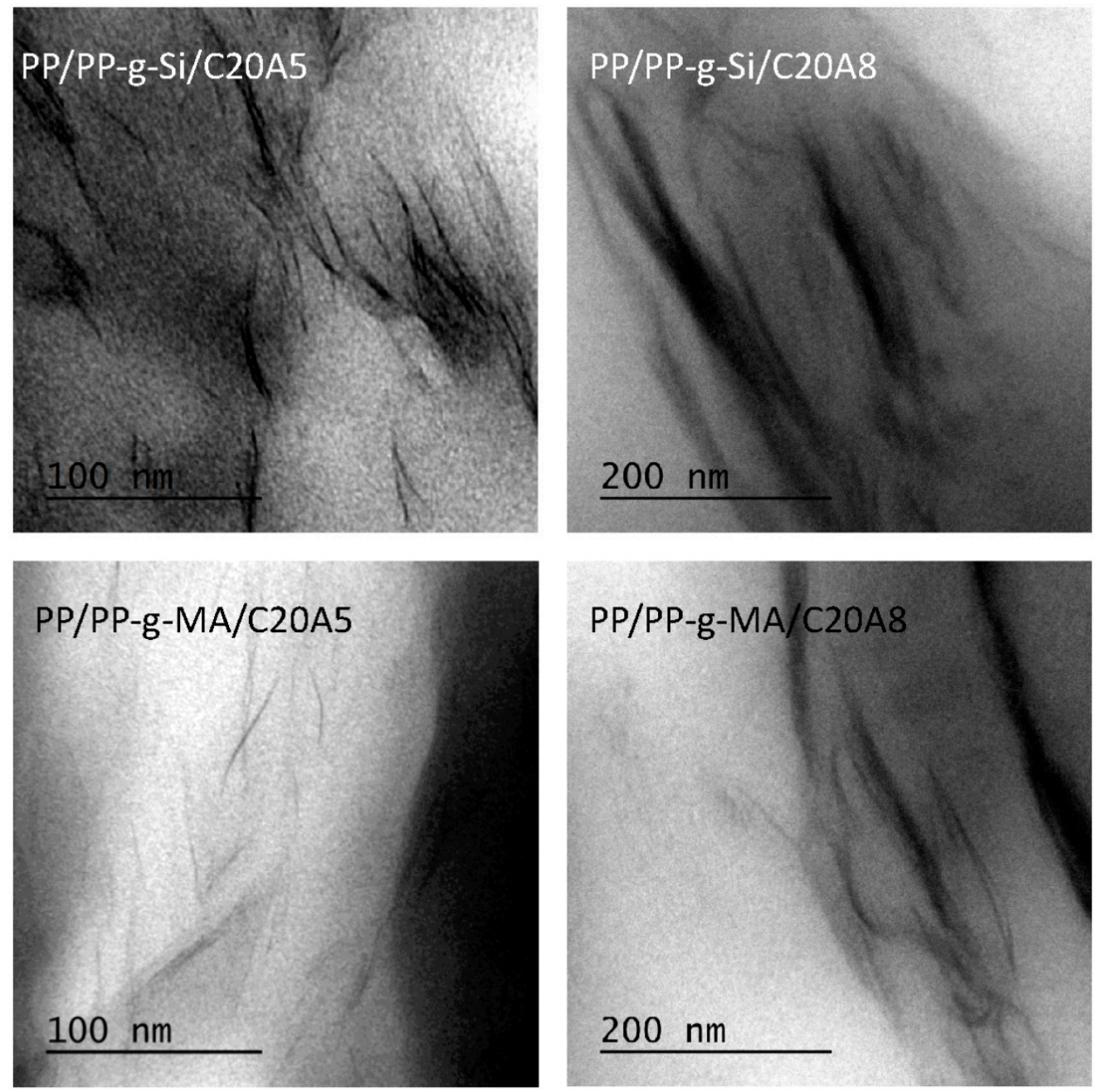

Figure 3. TEM micrographs of nanocomposites at 5 and $8 \mathrm{wt} \%$ clay contents.

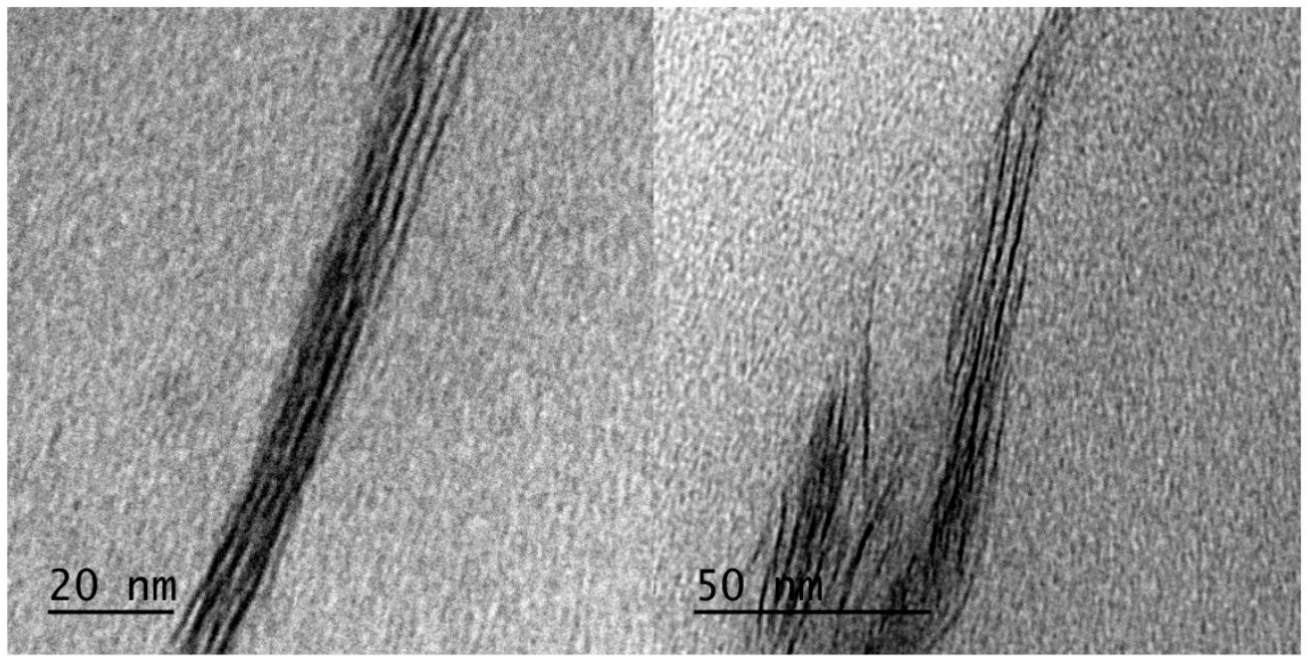

Figure 4. TEM micrographs of intercalated parts of PP/PP-g-Si/C20A3 nanocomposite. 


\subsection{Thermal Behaviour}

\subsubsection{Thermal Stability}

Figure 5 illustrates the TGA curves of virgin PP and nanocomposites based on silane and MA grafting PP. The detailed data corresponding to the decomposition temperatures at $5 \%\left(T_{5 \%}\right)$ and $T_{\max }$ are presented in Table 1. During thermal degradation, nanocomposites samples exhibited thermal decomposition temperature of about 5 to $14{ }^{\circ} \mathrm{C}$ at $T_{5 \%}$ and from 43 to $58{ }^{\circ} \mathrm{C}$ at $T_{\max }$, greater than the degradation temperature of the matrix, for the nanocomposites prepared with $\mathrm{PP}-\mathrm{g}-\mathrm{Si}$; whereas a variation from 8 to $32{ }^{\circ} \mathrm{C}$ was recorded at $\mathrm{T}_{5 \%}$ and 89 to $98^{\circ} \mathrm{C}$ at $T_{\max }$, greater than that of the matrix for nanocomposites prepared with PP-g-MA. In comparison to the matrix, samples without clay exhibited a decrease in both $T_{5 \%}$ and $T_{\max }$, with PP-g-Si as a compatibilizer and between $T_{5 \%}$ and $T_{\max }$ for PP-g-MA. It was noted also that PP-g-Si samples stabilized gradually from 340 to $1000{ }^{\circ} \mathrm{C}$ with a residue of 5 to $2 \mathrm{wt} \%$. Generally, the incorporation of clay into the polymer matrix enhances thermal stability by acting as a superior insulator and transport barrier to the volatile products generated during decomposition [18,19]. The remarkable improvement in thermal stability of PP-g-MA clay nanocomposites may depend on the state of dispersion of clay into the PP matrix and its content. The exfoliated structure of nanocomposites can easily lead to the char formation, which limited the diffusion of the thermo-oxidation products during decomposition. At lower clay loading ( $3 \mathrm{wt} \%)$, even if complete exfoliation is obtained, the amount of clay is not enough to provide the char formation. However, at higher loading (5 wt $\%$ ) of exfoliated nanocomposites, the highest thermal decomposition temperature was observed (about $98^{\circ} \mathrm{C}$ ). When the partial exfoliated structure was obtained, even if clay loading was high (case of $8 \mathrm{wt} \%$ ), thermal decomposition temperature was reduced because partial exfoliation does not lead to stabilization of materials despite the char formation. This would explain the differences observed between nanocomposites prepared with PP-g-MA and PP-g-Si.

Table 1. TGA data of pure PP, PP-g-Si, PP-g-MA, PP/PP-g-Si, PP/PP-g-MA, and all nanocomposites at different clay loadings.

\begin{tabular}{ccccc}
\hline Sample & \multicolumn{4}{c}{$T_{5 \%}\left({ }^{\circ} \mathrm{C}\right)$ Improvement $\left({ }^{\circ} \mathrm{C}\right) T_{\max }\left({ }^{\circ} \mathrm{C}\right)$ Improvement $\left({ }^{\circ} \mathrm{C}\right)$} \\
\hline PP & 265 & reference & 334 & reference \\
PP-g-Si & 247 & -18 & 316 & -18 \\
PP/PP-g-Si & 256 & -9 & 333 & -1 \\
PP/PP-g-Si/C20A3 & 270 & 5 & 377 & 43 \\
PP/PP-g-Si/C20A5 & 275 & 10 & 384 & 50 \\
PP/PP-g-Si/C20A8 & 279 & 14 & 392 & 58 \\
PP-g-MA & 249 & -16 & 327 & -7 \\
PP/PP-g-MA & 265 & 0 & 350 & 16 \\
PP/PP-g-MA/C20A3 & 273 & 8 & 423 & 89 \\
PP/PP-g-MA/C20A5 & 288 & 23 & 432 & 98 \\
PP/PP-g-MA/C20A8 & 297 & 35 & 427 & 93 \\
\hline
\end{tabular}



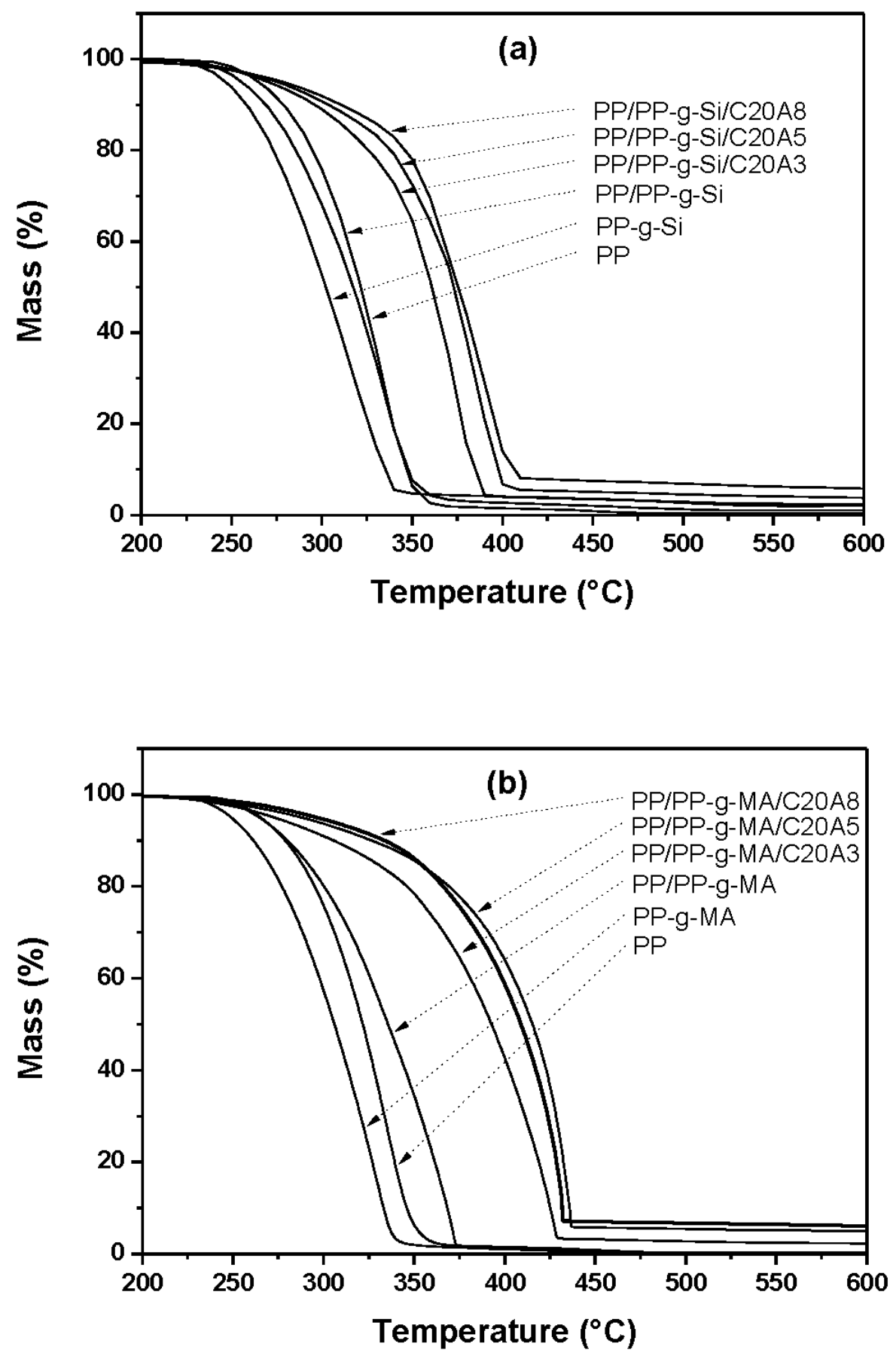

Figure 5. TGA curves of pure PP, PP-g-Si, PP-g-MA, PP/PP-g-Si, PP/PP-g-MA, and all nanocomposites at different clay contents.

\subsubsection{Melting and Crystallization Properties}

Generally, the properties of semicrystalline thermoplastic materials are strongly related to their internal microstructure and crystallinity, particularly the mechanical properties and thermal stability [20]. Thermal parameters of crystallization $\left(T_{c}\right)$ and melting $\left(T_{m}\right)$ temperatures, crystallization enthalpy $\left(\Delta H_{c}\right)$, heat of melting $\left(\Delta H_{m}\right)$, and the percentage of crystallinity $\left(X_{c}\right)$ are presented in Table 2 . The crystallization thermograms of neat PP, PP/PP-g-Si, and PP/PP-g-MA blends and nanocomposites are shown in Figure 6a. The results showed the differences between nanocomposites compatibilized with PP-g-Si and nanocomposites based on PP-g-MA. For neat PP, the crystallization temperature $\left(T_{c}\right)$ was observed at around $114.79{ }^{\circ} \mathrm{C}$. Furthermore, for the PP/PP-g-Si blend, $T_{c}$ increased by about $8{ }^{\circ} \mathrm{C}$, while PP/PP-g-MA showed no significant increase. In addition, the 
nanocomposites based on PP-g-Si presented a remarkable increase of $T_{\mathrm{c}}$ in comparison to those prepared with PP-g-MA (of about $17^{\circ} \mathrm{C}$ for $8 \mathrm{wt} \%$ of clay content). In the case of PP-g-MA, only the intercalated composition (with $8 \mathrm{wt} \%$ of clay) recorded an enhancement in the crystallization temperature. This increase is informative of a heterogeneous nucleation process in the presence of clay, which induced and facilitated the crystallization process of PP during cooling and was thus promoted by the presence of PP-g-Si, which acted as a nucleating agent during PP crystallization. This is also observed by the increase of crystallinity content of nanocomposites prepared with PP-gSi upon clay addition and the decrease for PP-g-MA nanocomposites (Table 2). From Figure 6b, neat PP exhibited a melting temperature at around $165.34^{\circ} \mathrm{C}$ and the binary blends and all nanocomposites showed a slight shift up to $3{ }^{\circ} \mathrm{C}$ in the melting point (Table 2). In summary, the differences in the crystallization characteristics of PP by the addition of clays are of crucial importance for understanding the mechanical properties of prepared nanocomposites.

Table 2. Summarized results of Differential scanning calorimetry (DSC) analysis of PP and its nanocomposites with different clay contents.

\begin{tabular}{cccccc}
\hline \multicolumn{7}{c}{$\left.\mathrm{Tc}^{\circ}{ }^{\circ} \mathrm{C}\right) \boldsymbol{\Delta H c}(\mathbf{J} / \mathbf{g}) \boldsymbol{T m}\left({ }^{\circ} \mathbf{C}\right) \boldsymbol{\Delta} \boldsymbol{H m}(\mathbf{J} / \mathbf{g}) \mathbf{X c}(\mathbf{\%})$} \\
\hline PP & 114.79 & 105.18 & 165.34 & 105.66 & 50.50 \\
PP/PP-g-Si & 122.31 & 108.84 & 165.34 & 108.66 & 51.92 \\
PP/PP-g-MA & 115.70 & 106.20 & 166.91 & 104.46 & 49.93 \\
PP/PP-g-Si/C20A3 & 127.25 & 103.20 & 167.01 & 105.60 & 52.03 \\
PP/PP-g-Si/C20A5 & 128.09 & 105.30 & 167.00 & 109.26 & 54.97 \\
PP/PP-g-Si/C20A8 & 131.08 & 102.36 & 168.60 & 104.82 & 54.46 \\
PP/PP-g-MA/C20A3 114.75 & 102.54 & 166.99 & 99.66 & 49.11 \\
PP/PP-g-MA/C20A5 114.89 & 101.58 & 166.12 & 97.62 & 49.14 \\
PP/PP-g-MA/C20A8 123.92 & 99.48 & 168.56 & 100.56 & 51.68 \\
\hline
\end{tabular}
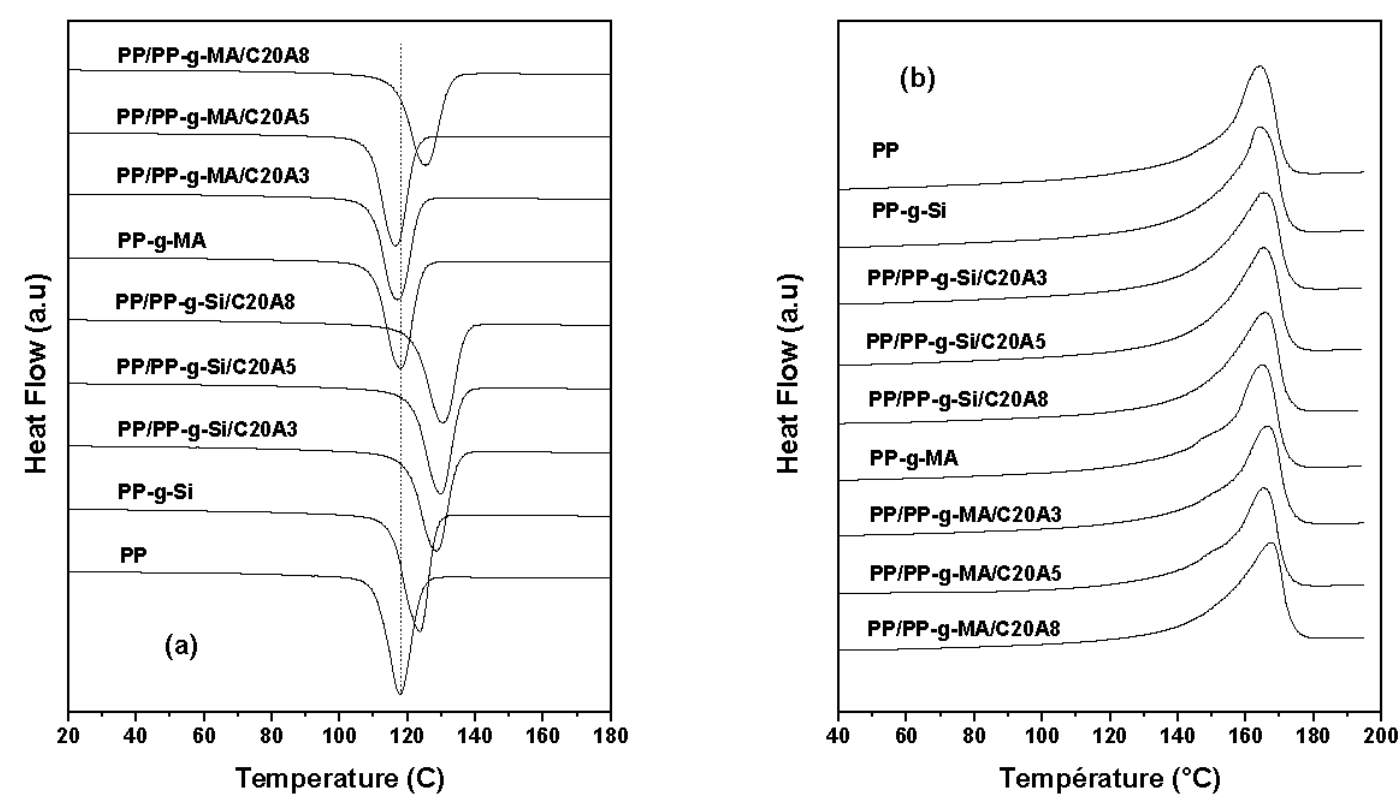

Figure 6. Crystallization thermograms (first cooling cycle) (a) and melting thermograms (second heating cycle) (b) of neat PP and its nanocomposites with various clay contents at $10^{\circ} \mathrm{C} / \mathrm{min}$ cooling and heating rates.

\subsection{Morphological Properties}

Linear viscoelastic properties are helpful in determining the strength of polymer layered silicate interactions and the structure-property relationship in nanocomposites at low frequency ranges [21]. Figure 7 presents the storage modulus, $G^{\prime}$, and complex viscosity, $\eta^{*}$, as a function of 
frequency for PP, PP-g-Si, PP-g-MA, PP/PP-g-Si, PP/PP-g-MA, and corresponding nanocomposites obtained with different clay loadings. Compared to PP, both modified PP showed lower complex viscosity. Atlow frequencies, the reduced melt viscosity of PP-g-Si was pronounced whereas the presence of PP-g-MA was found to be changed slightly. When the clay content was increased, a dramatic rise was noted in both of $G^{\prime}$ and $\eta^{*}$, leading to the plateau in the shear moduli and nonNewtonian behavior viscosity, for nanocomposites compatibilized with PP-g-MA, at low frequencies. For nanocomposites compatibilized with PP-g-Si, the storage modulus and complex viscosity behaviors were identical to those of the neat PP and increased progressively with the increase of clay content, keeping Newtonian behavior in viscosity. These noticeable differences were namely related to the difference in the state of dispersion of clay and to the interaction between components. In the presence of PP-g-Si, the alkylammonium surfactant/polymer interaction was favored, which led to intercalated/partial exfoliation since the silane end group grafted onto PP was ethyl, and polymer interacts with clay by Van der Waals interaction, promoting silicates' interactions. However, MA grafted onto PP is capable of hydrogen bonding with hydroxyl group of clay lamella, leading to better dispersion of those clay lamellas in matrix, as revealed by TEM observations and XRD analysis. It is noteworthy to mention that the bond energy of hydrogen bonds was lower than covalent bonds, but much higher than the bond energy of Van der Waals forces between two atoms. Consequently, when the clay content was increased, the extent of percolated network structures formed by exfoliated layers or stacks of intercalated layers, also called tactoids, increased [22]. When subjected to shear, individual lamellae and tactoids were beyond a critical volume fraction, incapable of rotating freely, and thus prevented from complete relaxation [23].
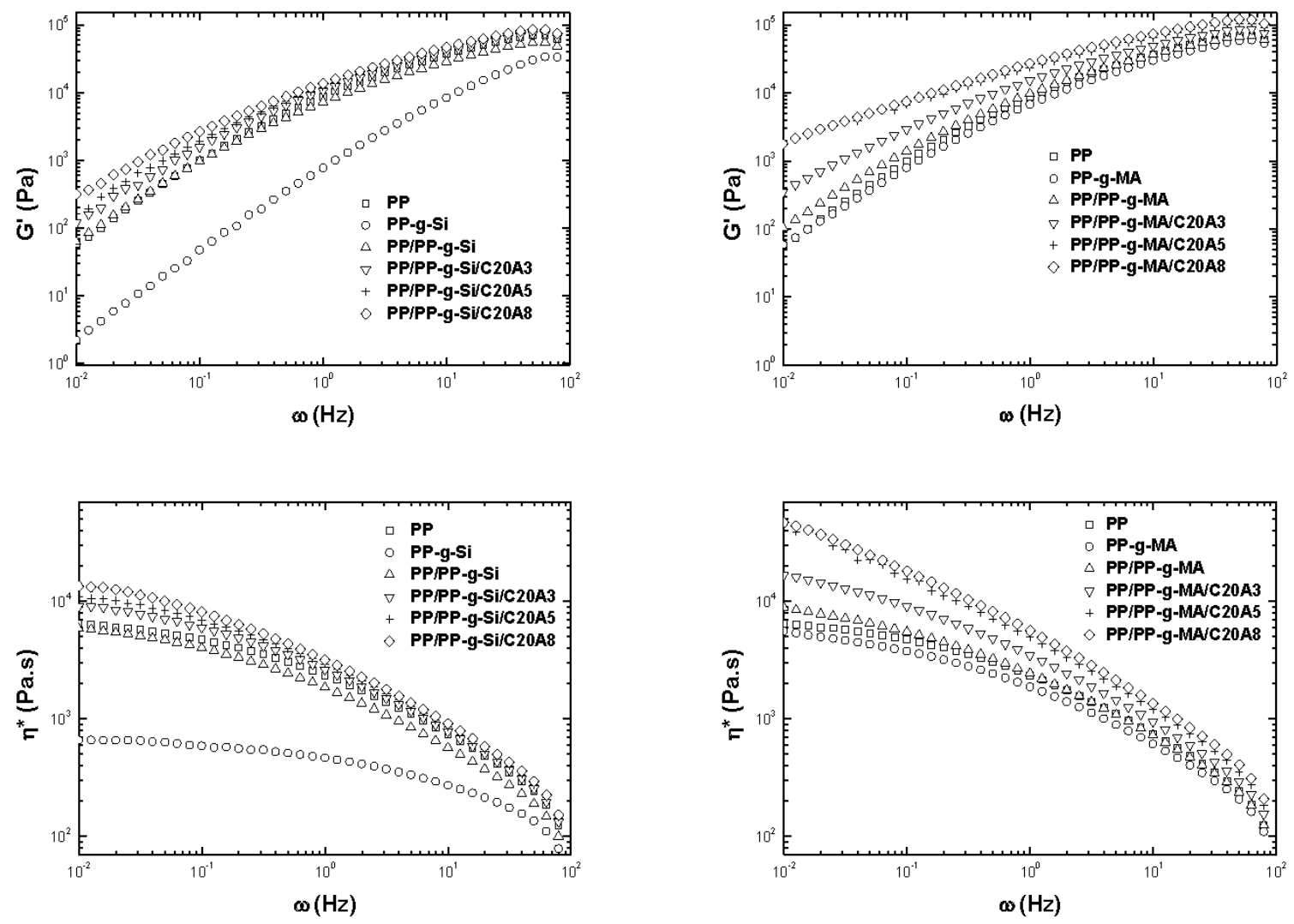

Figure 7. Elastic modulus, $G^{\prime}$, and complex viscosity, $\eta^{*}$, at $180{ }^{\circ} \mathrm{C}$ as a function of the frequency of pure PP, PP-g-Si, PP-g-MA, PP/PP-g-Si, PP/PP-g-MA, and all nanocomposites at different clay contents. 


\subsection{Tensile Properties}

Homogeneous dispersion of clay nanolayers in the polymer matrix is known to provide reinforcement via load transfer and deflection of cracks resulting from an applied load, due to its high aspect ratio and platelet structure. Interactions between exfoliated nanolayers with wide interfacial area and the surrounding polymer matrix can lead to higher mechanical properties [24]. The tensile properties of samples were determined to establish the effect of compatibilizing agents and the clay content. Figure 8 shows the Young's modulus of PP, PP-g-Si, PP-g-MA, PP/PP-g-Si, PP/PP-g-MA, and nanocomposites obtained with different clay contents. It can be seen from the results that all nanocomposites achieved a higher modulus than neat PP, while, as expected, PP-g-Si and PP-g-MA decreased. This reduction is attributed to the chain scission resulting from chemical treatment of PP with peroxides, lowering PP molecular weight and, consequently, the mechanical properties. Clear differences in the tensile properties of the nanocomposites with different types of compatibilizer are shown. The property enhancements of nanocomposites became more significant with the incorporation of the PP-g-MA compared to the PP-g-Si compatibilizer. As mentioned above, the state of dispersion, the level of interaction strength between the organoclay and polymer, and the rheological properties of nanocomposites containing PP-g-MA were much enhanced compared to compositions based on PP-g-Si.

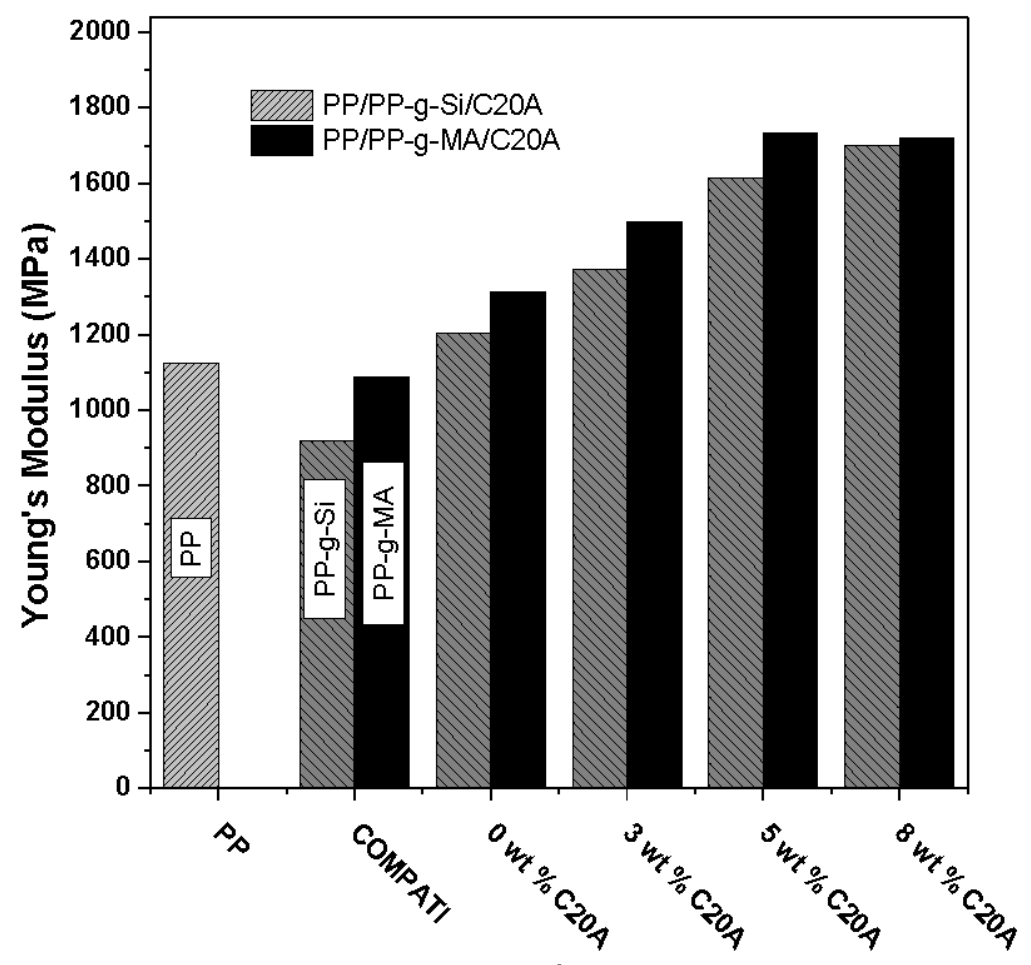

Figure 8. Mechanical properties of pure PP and their corresponding nanocomposites at different clay contents.

\section{Materials and Methods}

\subsection{Materials}

The PP used throughout this investigation was a homopolymer, PP 5032E1, commercialized by Exxon Mobil chemical. Dicumyl peroxide (DCP, 98\%), vinyltriethoxysilane (VTES, 97\%), and maleic anhydride (MA, 99\%) were purchased from Sigma Aldrich. The filler was layered silicate, chemically modified with a dimethyl, dehydrogenated tallow quaternary ammonium salt (Cloisite 20A, C20A) purchased from southern Clay products Inc. Texas, TX, USA 


\subsection{Grafting Procedure}

PP-g-Si. PP powder (50 g) was tumble mixed with a solution of DCP (0.2 wt $\%)$ in VTES (5 wt\%) for $20 \mathrm{~min}$. The grafting reaction was carried out in a Haake Polylab rheometer equipped with a Rheomix mixing chamber and roller rotor. The mixture was reacted for $5 \mathrm{~min}$ at $180{ }^{\circ} \mathrm{C}$ at a rotation speed of $60 \mathrm{rpm}$, giving PP-g-Si.

$P P-g-M A$. PP powder $(50 \mathrm{~g})$ was tumble mixed with a solution of chloroform and DCP $(0.2 \mathrm{wt} \%)$ in MA ( $5 \mathrm{wt} \%)$ for $20 \mathrm{~min}$. The grafting reaction was carried out in the same condition as PP-g-Si. The mixture is denoted as PP-g-MA

\subsection{Nanocomposites' Preparation}

Melt mixing of PP/PP-g-Si (or PP-g-MA)/C20A composites were carried out using contrarotating twin screw micro extruder Minilab II. PP, PP-g-Si (or PP-g-MA) and clay were tumble mixed and introduced in the hopper. 500 ppm of irganox-1010, 1000 ppm of Irgafos-168 and 600 ppm of calcium stearate were added after $5 \mathrm{~min}$ of mixing time for 2 min to stabilize the PP-g-Si for nanocomposites' samples. The temperature, screw speed, and residence time were fixed at $180{ }^{\circ} \mathrm{C}$, $60 \mathrm{rpm}$, and $5 \mathrm{~min}$ respectively. The composition of PP/PP-g-Si (or PP-g-MA) was kept at 90 and $10 \mathrm{wt} \%$ for all blends and the clay concentrations were fixed at 3, 5, and $8 \mathrm{wt} \%$. The notation used in the following for defining the blend composition is PP/PP-g-Si/ (or PP-g-MA)/C20A, and is expressed in weight fraction.

\subsection{Characterizations and Techniques}

\section{Characterization of Functionalized PP}

PP-g-Si samples for graft content analysis were purified form residual VTES by dissolving in hot refluxing xylene stabilized in 100 ppm of 2,6-di-t-butyl-4-methylphenol (BHT), precipitating from acetone and dried under vacuum at $60^{\circ} \mathrm{C}$. FTIR spectra were obtained using an ABB Bomem FTLA 2000-102 FTIR instrument, in transmission mode (Figure 1). Grafted VTES contents were calculated from FTIR integrations of the $1064-1094 \mathrm{~cm}^{-1}$ absorbance of the silane relative to $422-496 \mathrm{~cm}^{-1}$ internal standard region originating from PP. A calibration curve for determining the graft content was obtained by using known mixtures of PP and unreactive silane as standards. Based on the calculation, the grafted VTES content was determined to be $0.51 \mathrm{wt} \%$.

PP-g-MA samples were purified from residual MA by dissolving in hot refluxing xylene, precipitating from acetone, and dried under vacuum at $60^{\circ} \mathrm{C}$. Grafted MA contents were calculated from FTIR integrations of the area derived from $1754-1808 \mathrm{~cm}^{-1} \mathrm{C}=\mathrm{O}$ anhydride absorbance relative to a $422-496 \mathrm{~cm}^{-1}$ internal standard region originating from PP. Calibration standards for this FTIR method were prepared by melt mixing known quantities of 1-dodecenylsuccinic anhydride to purified starting material. The grafted MA content was determined to be $0.33 \mathrm{wt} \%$.

\subsection{Morphology Studies}

The structure of layered silicates and the morphology of composites were analyzed by X-ray scattering, using a Bruker D8 AXS X-ray diffractometer. Diffraction spectra were obtained over a $2 \theta$ range of $1-10^{\circ}$ and the interlayer spacing (doo1) was calculated using the Bragg equation: $n \lambda=2 d \mathrm{hksin} \theta$, where $\lambda$ is the wavelength of radiation ( $\mathrm{Cu}-\mathrm{K} \alpha$ radiation of $1.542 \AA)$. The samples were prepared as discs of $25 \mathrm{~mm}$ in diameter and $1.5 \mathrm{~mm}$ in thickness, by compression molding at $180{ }^{\circ} \mathrm{C}$. Each measurement was repeated four times, on two different surfaces. TEM observations were performed with a Philips CM200 TEM microscope operating at $200 \mathrm{kV}$ and coupled to an energy dispersive (EDS) microprobe analyzer (EDAX DX-4). Ultrathin sections (ca. $100 \mathrm{~nm}$ ) were cut into pieces with an ultramicrotome (Leica EM UC7) using a diamond knife at room temperature. The pieces were put on a carbon-coated copper TEM grid and then imaged with a TEMapparatus. 


\subsection{Thermal Properties (TGA and DSC)}

Thermogravimetric analyses (TGA) were conducted by using a Q500 TA instrument thermoanalyzer. The specimens with weights of about $15 \mathrm{mg}$ were heated from room temperature to $1000{ }^{\circ} \mathrm{C}$ at a linear heating rate of $10^{\circ} \mathrm{C} / \mathrm{min}$. All runs were performed in an air atmosphere at a flow of $60 \mathrm{~mL} / \mathrm{min}$.

The melting and crystallization behaviors of samples (approximately $12 \mathrm{mg}$ ) were analyzed by the usage of the conventional differential scanning calorimeter, DSC-Q100, from TA Instrument. The heating program was as follows:

- First heating scan: Heating from 25 to $200^{\circ} \mathrm{C}$ with a rate of $10^{\circ} \mathrm{C} / \mathrm{min}$;

- $\quad$ keeping the sample at $200^{\circ} \mathrm{C}$ for $5 \mathrm{~min}$ to erase the thermal history;

- first cooling scan: Cooling to $-60^{\circ} \mathrm{C}$ with a rate of $10^{\circ} \mathrm{C} / \mathrm{min}$; and

- second heating scan: Heating from -60 to $200{ }^{\circ} \mathrm{C}$ with a rate of $10{ }^{\circ} \mathrm{C} / \mathrm{min}$.

All DSC runs were carried out under a nitrogen atmosphere. The melting and crystallization peaks were obtained from the second heating and first cooling scans, respectively. The degree of crystallinity $\left(X_{c}\right)$ was determined by using the following equation:

$$
X_{\mathrm{c}}(\%)=\frac{\Delta H_{m}}{(1-\phi) \Delta H_{m}^{0}} \times 100
$$

where $\phi$ was the clay content, $\Delta H_{m}$ was the apparent melting enthalpy, and $\Delta H_{m}^{0}$ was the extrapolated value of the enthalpy corresponding to the melting of $100 \%$ crystalline PP $\left(\Delta H_{m}^{0}=209.2 \mathrm{~J} / \mathrm{g}\right.$ [25]).

\subsection{Rheological Measurements}

Rheological characterizations were performed in small-amplitude oscillatory shear flow on an ARES- LS rheometer, using parallel plate geometry with a $25 \mathrm{~mm}$ in diameter and $1 \mathrm{~mm}$ gap. Test specimens were prepared by compression molding at $180{ }^{\circ} \mathrm{C}$. The storage and loss moduli, $\mathrm{G}^{\prime}$ and $G^{\prime \prime}$, and complex shear viscosity were measured over a frequency $(\omega)$ range of $0.01-100 \mathrm{~Hz}$ at $180^{\circ} \mathrm{C}$. All measurements were carried out in the liner viscoelastic regime by imposing a strain of $0.05 \%$ as assessed by preliminary strain sweep tests.

Mechanical properties. Specimens for mechanical testing were prepared by compression molding at $180^{\circ} \mathrm{C}$ using a carver press. Tensile properties were measured using an Instron 8810 S universal tester machine, according to ASTM D638, at crosshead speeds of $10 \mathrm{~mm} / \mathrm{min}$. At least 5 specimens were tested for each sample and average values were reportedhere.

\section{Conclusions}

PP/clay nanocomposites based on PP-g-Si and PP-g-MA as compatibilizing agents were prepared by melt compounding. The presence of compatibilizers impacted the interaction polymer/filler, therefore inducing the morphology change during the processing of nanocomposites. The use of PP-g-Si led to partial exfoliated/intercalated structures whereas PP-g-MA resulted in an exfoliated structure at lower clay contents ( $\leq 5 \mathrm{wt} \%$ ) and the coexistence of an exfoliated and intercalated structure at $8 \mathrm{wt} \%$. The improvement in properties is related to the polymer-clay characteristics, the level of interaction between the clay and polymer, and the obtained morphology. From thermal analysis, the presence of PP- g-Si induced an increase in the crystallization temperature and crystallinity of nanocomposites upon clay addition in comparison to PP-g-MA. An increase in thermal stability was also observed in both compatibilized nanocomposites. A higher value $\left(98^{\circ} \mathrm{C}\right)$ was obtained in $5 \mathrm{wt} \%$ of clay with PP-g-MA. The increase of clay content significantly changed the rheological behavior of nanocomposites at low frequency regions, reaching a pseudo-solid like behavior. Rheological measurement also informed about the state of clay dispersion in the polymer matrix. Significant mechanical reinforcement was also obtained and PP-g-MA showed a better Young's modulus. 
Author Contributions: H.R. conceived and designed the experiments; H.R. performed the experiments; H.R. and K.E.M. analyzed the data; K.E.M. contributed reagents/materials/analysis tools; H.R. wrote the paper. H.A.O. improve the discussion and edit the paper.

Funding: This work was funded by MAScIR-Moroccan Foundation for Advanced Science, Innovation and Research.

Acknowledgments: The support from Hassan II Academy of Science and Technology, Rabat, Morocco is also greatly appreciated. The authors are grateful for the financial support from EC (project REGPOT ALNANOFUNC grant number REGPOTCT-2011-285895-Al-NANOFUNC-FP7).

Conflicts of Interest: We declare that this manuscript is original, has not been reported before, and is not currently being considered elsewhere. We also confirm that there is no known conflict of interest regarding this manuscript and its publication. The manuscript has been approved by all named authors.

\section{References}

1. Sinha Ray, S.; Okamoto, M. Polymer/layered silicate nanocomposites: A review from preparation to processing. Prog. Polym. Sci. 2003, 28, 1539-1641.

2. Lertwimolnun, W.; Vergnes, B. Influence of compatibilizer and processing conditions on the dispersion of nanoclay in a polypropylene matrix. Polymer 2005, 46, 3462-3471.

3. Ding, C.; Jia, D.; He, H.; Guo, B.; Hong, H. How organo-montmorillonite truly affects the structure and properties of polypropylene. Polym. Test. 2005, 24, 94-100.

4. López-Quintanilla, M.L.; Sánchez-Valdés, S.; Ramos de Valle, L.F.; Medellín-Rodríguez, F.J. Effect of some compatibilizing agents on clay dispersion of polypropylene-clay nanocomposites. J. Appl. Polym. Sci. 2006, 100, 4748-4756.

5. Sengupta, S.S.; Parent, J.S.; McLean, J.K. Radical-mediated modification of polypropylene: Selective grafting via polyallyl coagents. J. Polym. Sci. Part A Polym. Chem. 2005, 43, 4882-4893.

6. Bailly, M.; Kontopoulou, M. Preparation and characterization of thermoplastic olefin/nanosilica composites using a silane-grafted polypropylene matrix. Polymer 2009, 50, 2472-2480.

7. Lu, H.; Hu, Y.; Li, M.; Chen, Z.; Fan, W. Structure characteristics and thermal properties of silane-graftedpolyethylene/clay nanocomposite prepared by reactive extrusion. Compos. Sci. Technol. 2006, 66, 3035-3039.

8. Sánchez-Valdes, S.; Méndez-Nonell, J.; Medellín-Rodríguez, F.J.; Ramírez-Vargas, E.; Martínez-Colunga, J.G.; Soto-Valdez, H.; et al. Effect of PEgMA/amine silane compatibilizer on clay dispersion of polyethylene- clay nanocomposites. Polym. Bull. 2009, 63, 921-933.

9. Dal Castel, C.; Pelegrini, T.; Barbosa, R.V.; Liberman, S.A.; Mauler, R.S. Properties of silane grafted polypropylene/montmorillonite nanocomposites. Compos. Part A Appl. Sci. Manuf. 2010, 41, 185-191.

10. Liaw, W.-C.; Huang, P.-C.; Chen, C.-S.; Lo, C.-L.; Chang, J.-L. PPgMA/APTS compound coupling compatabilizer in PP/clay hybrid nanocomposite. J. Appl. Polym. Sci. 2008, 109, 1871-1880.

11. Russell, K.E. Free radical graft polymerization and copolymerization at higher temperatures. Prog. Polym. Sci. 2002, 27, 1007-1038.

12. Santos, K.S.; Liberman, S.A.; Oviedo, M.A.S.; Mauler, R.S. Polyolefin-based nanocomposites: The effect of organosilane on organoclay dispersion. J. Mater. Sci. 2013, 49, 70-78.

13. Nachtigall, S.M.B.; Stedile, F.C.; Felix, A.H.O.; Mauler, R.S. Polypropylene Functionalization with Vinyltriethoxysilane. J. Appl. Polym. Sci.1998, 72, 1313-1319.

14. El Mabrouk, K.; Parent, J.S.; Chaudhary, B.I.; Cong, R. Chemical modification of PP architecture: Strategies for introducing long-chain branching. Polymer 2009, 50, 5390-5397.

15. Rogers, K.; Takacs, E.; Thompson, M.R. Contact angle measurement of select compatibilizers for polymersilicate layer nanocomposites. Polym. Test. 2005, 24, 423-427.

16. Wiederrecht, G.P. Handbook of Nanofabrication; Boston: Elsevier: Amsterdam, The Netherlands, 2010.

17. Giannelis, E.P.; Krishnamoorti, R.; Manias, E. Polymer-Silicate Nanocomposites: Model Systems for Confined Polymers and Polymer Brushes. Adv. Polym. Science. 1999, 138, 107-147.

18. Devalckenaere, M.; Je, R.; Dubois, P.; Kubies, D. Poly (1-caprolactone)/clay nanocomposites prepared by melt intercalation: Mechanical, thermal and rheological properties. Polymer 2002, 43, 4017-4023.

19. Zhu, J.; Morgan, A.B.; Lamelas, F.J.; Wilkie, C.A. Fire Properties of Polystyrene-Clay Nanocomposites. Chem. Mater. 2001, 13, 3774-3780.

20. Bureau, M.N.; Denault, J.; Cole, K.C.; Enright, G.D. The role of crystallinity and reinforcement in the mechanical behavior of polyamide-6/clay nanocomposites. Polym. Eng. Sci. 2002, 42, 1897-1906. 
21. Ray, S.S. Rheology of Polymer / Layered Silicate Nanocomposites. J. Ind. Eng. Chem. 2006, 12, 811-842.

22. Kim, D.H.; Fasulo, P.D.; Rodgers, W.R.; Paul, D.R. Structure and properties of polypropylene-based nanocomposites: Effect of PP-g-MA to organoclay ratio. Polymer 2007, 48, 5308-5323.

23. Hyun, Y.H.; Lim, S.T.; Choi, H.J.; Jhon, M.S. Rheology of Poly(ethylene oxide)/Organoclay Nanocomposites. Macromolecules 2001, 34, 8084-8093.

24. Liang, J.-Z. Toughening and reinforcing in rigid inorganic particulate filled poly(propylene): A review.J. Appl. Polym. Sci. 2002, 83, 1547-1555.

25. Devalckenaere, M.; Je, R.; Dubois, P.; Kubies, D. Poly (1-caprolactone)/clay nanocomposites prepared by melt intercalation: Mechanical, thermal and rheological properties. Polymer 2002, 43, 4017-4023.

(C) 2018 by the authors. Licensee MDPI, Basel, Switzerland. This article is an open access article distributed under the terms and conditions of the Creative Commons Attribution (CC BY) license (http://creativecommons.org/licenses/by/4.0/). 